\title{
A new united atom force field for $\alpha$-olefins
}

\author{
Shyamal K. Nath, ${ }^{\text {a) }}$ Brian J. Banaszak, and Juan J. de Pablo ${ }^{\text {b) }}$ \\ Department of Chemical Engineering, University of Wisconsin, Madison, Wisconsin 53706
}

(Received 3 April 2000; accepted 1 December 2000)

\begin{abstract}
A new united-atom force field is proposed for $\alpha$-olefins. The proposed force field is used to study vapor-liquid equilibria for pure components and binary mixtures. Results of phase equilibria simulations are found to be in good agreement with available experimental data. (c) 2001 American Institute of Physics. [DOI: 10.1063/1.1343487]
\end{abstract}

\section{INTRODUCTION}

Recent advances in simulation techniques have made calculation of phase equilibria of complex systems possible from atomistic models of intermolecular interactions. From simple Lennard-Jones ${ }^{1}$ fluids to fairly realistic models of molecules, coexistence (e.g., vapor-liquid) properties can now be simulated with unprecedented levels of accuracy. Simulation of phase equilibria for long, articulated molecules has also become possible with the development of configurational-bias and expanded-ensemble techniques. ${ }^{2-4}$

Coexistence properties and, in particular, orthobaric densities, provide a stringent test of the accuracy of a force field. Furthermore, the wide availability of vapor-liquid equilibrium experimental data make simulations of coexistence properties an attractive means of optimizing or refining existing force fields. Recently, several united-atom force fields have appeared in the literature which can accurately describe coexistence curves for normal alkanes ${ }^{5-7}$ and branched alkanes. ${ }^{8}$ In previous work, we have developed united atom force fields for both normal alkanes ${ }^{5}$ and branched alkanes ${ }^{8}$ [the Nath, Escabedo, and de Pablo revised (NERD) force field] which are capable of reproducing experimental phase equilibrium data. Here, we extend the NERD force field to the $\alpha$-olefins.

Knowledge of phase-equilibria for alkane and other olefins is important for both the polymer and the petro-chemical industries. Over the last few years, with the development of novel processes and products, the use of $\alpha$-olefins as reactants, intermediates, or end products has increased significantly. Accurate prediction of thermodynamic properties and phase equilibria of the $\alpha$-olefins and their mixtures is important for optimal design of such process and products. Industrial applications have often relied on empirical correlations and semitheoretical equations of state to predict phase equilibria; unfortunately the predictive capabilities of commonly used equations of state of mixtures have recently been called into question. ${ }^{9,10}$ Given the chemical simplicity of alkanes and olefins, molecular simulations could provide a useful complement or alternative in the study and description of the phase behavior of these systems.

\footnotetext{
${ }^{\text {a) }}$ Current address: Molecular Simulations Inc., 9685 Scranton Road, San Diego, CA 92121.

b) Author to whom correspondence should be addressed. Electronic mail: depablo@engr.wisc.edu
}

To our knowledge, only two studies have proposed united atom force fields for $\alpha$-olefins. In the first of these, Jorgensen et al. ${ }^{11}$ proposed a model for 1-butene. These authors examined the liquid density, boiling point, heat capacity, thermal expansion coefficient and compressibility at $25^{\circ} \mathrm{C}$. The second, proposed recently by Spyriouni et al., ${ }^{12}$ is based on pure-component phase equilibrium data for $\alpha$-olefins ranging from 1-butene to 1-octene. Although a force field based on pure-component phase equilibrium already exists for $\alpha$-olefins, our search for a new set of parameters is motivated by two reasons; first, based on the level of agreement with pure component experimental phase equilibrium data and Spyriouni et al. predictions, we felt that there is still room for model improvement, and, second, we are interested in force fields for $\alpha$-olefins that are compatible with our existing NERD force field. The new force field would be used to obtain mixture properties of $\alpha$-olefins with other alkanes.

\section{MODELS AND SIMULATION DETAILS}

As mentioned earlier, a united-atom representation of the alkanes and the alkenes is adopted throughout this work (e.g., $\mathrm{CH}_{3}, \mathrm{CH}_{2}$, and $\mathrm{CH}$ groups are described by single interaction sites). For simple $n$-alkanes, we use the NERD force field. ${ }^{5}$ Lennard-Jones parameters for the alkenes are obtained by slight modifications of those for $n$-alkanes. As for the $n$-alkanes ${ }^{5}$ and the branched alkanes, ${ }^{8}$ we decided to adopt the same $\mathrm{CH}_{2}$ parameters when $\mathrm{CH}_{2}$ is not an end site $\left(\mathrm{CH}_{2}\right.$ with $s p^{3}$ hybridization). Since the alkenes and the $n$-alkanes are similar, except the slight difference in one of the end groups, we expect that using the same $\mathrm{CH}_{2}\left(s p^{3}\right)$ parameter will result in a model for alkenes which, for pure components, is as accurate as the NERD model is for $n$-alkanes.

For $\mathrm{CH}$ sites, we adopt independent parameters for the alkenes as for the branched alkanes because the two cases have different hybridization. For alkenes, $\mathrm{CH}$ sites have $s p^{2}$ hybridization and for branched alkanes, $\mathrm{CH}$ sites have $s p^{3}$ hybridization.

An independent set of parameters for end $\mathrm{CH}_{2}\left(s p^{2}\right)$ groups is required for ethylene; for the other alkenes the parameters for the end $\mathrm{CH}_{2}\left(s p^{2}\right)$ groups are the same. Similarly, an independent set of parameters for the end $\mathrm{CH}_{3}\left(s p^{3}\right)$ site is required for propene; for the other alkenes, parameters 
TABLE I. Intramolecular potential energy functions.

Bond stretching potential

$V(r) / k_{B}=\frac{K_{r}}{2}\left(r-b_{\text {eq }}\right)^{2}$

$K_{r}=96500 \mathrm{~K} / \AA^{2}$

$b_{\text {eq }}=1.54 \AA(\mathrm{C}-\mathrm{C})$

$b_{\text {eq }}=1.34 \AA(\mathrm{C}=\mathrm{C})$

Bond bending potential

$V(\theta) / k_{B}=\frac{K_{\theta}}{2}\left(\theta-\theta_{\text {eq }}\right)^{2}$

$K_{\theta}=62500 \mathrm{~K} / \mathrm{rad}^{2}$

$\theta_{\mathrm{eq}}=114.0^{\circ}(\mathrm{C}-\mathrm{C}-\mathrm{C})$

$\theta_{\mathrm{eq}}=124.0^{\circ}(\mathrm{C}-\mathrm{C}=\mathrm{C})$

Torsional potential

$V(\phi) / k_{B}=V_{0}+V_{1}(1+\cos \phi)+V_{2}(1-\cos 2 \phi)+V_{3}(1+\cos 3 \phi)$

$\mathrm{C}-\mathrm{C}-\mathrm{C}-\mathrm{C}$

$V_{0}=0$

$V_{2}=-68.19 \mathrm{~K}$

$V_{1}=355.04 \mathrm{~K}$

$\mathrm{C}-\mathrm{C}-\mathrm{C}=\mathrm{C}$

$V_{0}=47.97 \mathrm{~K}$

$V_{2}=-109.71 \mathrm{~K}$

$V_{3}=791.32 \mathrm{~K}$

$V_{1}=86.31 \mathrm{~K}$

$V_{3}=282.08 \mathrm{~K}$

for the end $\mathrm{CH}_{3}\left(s p^{3}\right)$ site are the same. Tables I and II provide a complete list of intra and intermolecular NERD force field parameters for both normal alkanes and alkenes. In all calculations, a cutoff radius of $10.0 \AA$ was employed for Lennard-Jones interactions and standard tail corrections were implemented. As in our previous work, we use simple Lorentz-Berthelot combining rules for the interaction parameters between unlike pairs

$$
\begin{aligned}
\sigma_{i j} & =\frac{1}{2}\left(\sigma_{i i}+\sigma_{j j}\right), \\
\epsilon_{i j} & =\sqrt{\epsilon_{i i} \epsilon_{j j}} .
\end{aligned}
$$

All coexistence-curve simulations were conducted by means of an expanded Gibbs ensemble formalism. ${ }^{4}$ All simulations were supplemented by configurational-bias moves. For pure components, all simulations were conducted at con-

TABLE II. Intermolecular potential energy functions.

Nonbonded interaction potential:

$V(r)=4 \epsilon\left[\left(\frac{r}{\sigma}\right)^{12}-\left(\frac{r}{\sigma}\right)^{6}\right]$

All Molecules, when $\mathrm{CH}_{n}$ is a terminal group Ethane $\left(\mathrm{CH}_{3}-\mathrm{CH}_{3}\right)$

$\sigma_{\mathrm{CH}_{3}\left(s p^{3}\right)}=3.825 \AA$

Ethylene $\left(\mathrm{CH}_{2}=\mathrm{CH}_{2}\right)$

$\sigma_{\mathrm{CH}_{2}\left(s p^{2}\right)}=3.79 \AA$

Propane $\left(\mathrm{CH}_{3}-\mathrm{CH}_{2}-\mathrm{CH}_{3}\right)$

$\sigma_{\mathrm{CH}_{3}\left(s p^{3}\right)}=3.857 \AA$

Propene $\left(\mathrm{CH}_{2}=\mathrm{CH}-\mathrm{CH}_{3}\right)$

$\sigma_{\mathrm{CH}_{3}\left(s p^{3}\right)}=3.85 \AA$

$\sigma_{\mathrm{CH}_{2}\left(s p^{2}\right)}=3.72 \AA$

$\sigma_{\mathrm{CH}\left(s p^{2}\right)}=3.77 \AA$

Molecules longer than Propane/Propen

$\sigma_{\mathrm{CH}_{2}\left(s p^{2}\right)}=3.72 \AA$

$\sigma_{\mathrm{CH}_{3}\left(s p^{3}\right)}=3.91 \AA$

$$
\begin{aligned}
\epsilon_{\mathrm{CH}_{3}\left(s p^{3}\right)} & =100.6 \mathrm{~K} \\
\epsilon_{\mathrm{CH}_{2}\left(s p^{2}\right)} & =84.7 \mathrm{~K} \\
\epsilon_{\mathrm{CH}_{3}\left(s p^{3}\right)} & =102.6 \mathrm{~K} \\
\epsilon_{\mathrm{CH}_{3}\left(s p^{3}\right)} & =100.0 \mathrm{~K} \\
\epsilon_{\mathrm{CH}_{2}\left(s p^{2}\right)} & =92.5 \mathrm{~K} \\
\epsilon_{\mathrm{CH}\left(s p^{2}\right)} & =46.0 \mathrm{~K} \\
\epsilon_{\mathrm{CH}_{2}\left(s p^{2}\right)} & =92.5 \mathrm{~K} \\
\epsilon_{\mathrm{CH}_{3}\left(s p^{3}\right)} & =104.0 \mathrm{~K}
\end{aligned}
$$

All Molecules, when $\mathbf{C H}_{n}$ is not a terminal group

$\sigma_{\mathrm{CH}_{2}\left(s p^{3}\right)}=3.93 \AA$

$\sigma_{\mathrm{CH}\left(s p^{2}\right)}=3.77 \AA$

$\sigma_{\mathrm{CH}\left(s p^{3}\right)}=3.85 \AA$

$\epsilon_{\mathrm{CH}_{2}\left(s p^{3}\right)}=45.8 \mathrm{~K}\left(-\mathrm{CH}_{2}-\right)$

$\epsilon_{\mathrm{CH}\left(s p^{2}\right)}=46.0 \mathrm{~K}(=\mathrm{CH}-)$

$\epsilon_{\mathrm{CH}\left(s p^{3}\right)}=39.7 \mathrm{~K}$ (CH is branch point)

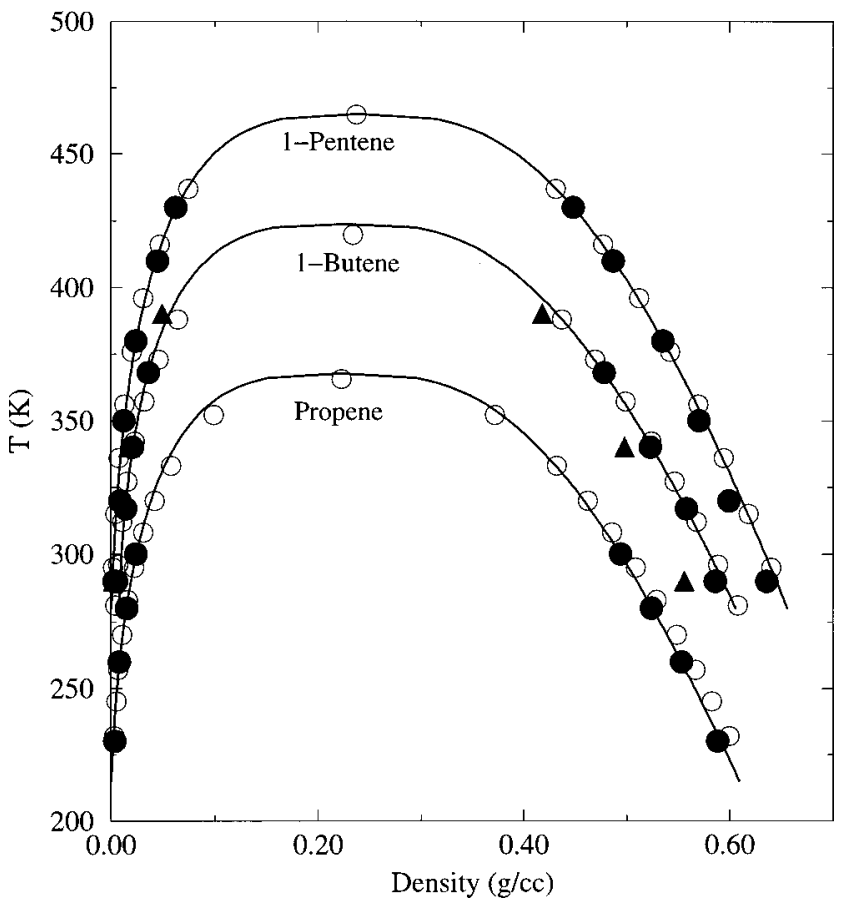

FIG. 1. Orthobaric densities for small alkenes. The filled circles are simulation results from present work, the filled triangles are from Spyriouni et al. (Ref. 12), open symbols are experimental results (Ref. 17), and the lines show an Ising fit to the simulation points. The error bars near the critical point are less than three times the size of the symbols. Error bars at lower temperatures are less than twice the size of the symbols.

stant volume and temperature. For mixtures, all simulations were conducted at constant pressure and temperature. Vapor pressures for pure components where calculated by evaluating the pressure from constant number of molecules, volume, and temperature molecular dynamics (NVT-MD) simulations at the coexistent vapor densities obtained from coexistence calculations. For detailed accounts of the simulation techniques, readers are referred to the original publications. ${ }^{4,13}$

In all our simulations, molecules were displaced by means of a hybrid Monte Carlo (HMC) procedure. ${ }^{14,15}$ Within the HMC procedure, five molecular dynamics steps were used to generate a global trial Monte Carlo move. A time step of 2 ps was used for the molecular-dynamics moves, as part of the HMC procedure.

The sizes of the systems investigated for purecomponent simulations ranged from 400 molecules for 1-propene to 100 molecules for 1-octene. For simulations of mixtures, the sizes of the systems ranged from 200 to 300 molecules for mixtures of alkenes and hexatriacontane. Equilibrium averages were collected for at least $2 \times 10^{7}$ simulation steps, of which about 5\% were volume moves, 5\%-10\% were HMC moves and the rest were transfer moves.

\section{RESULTS AND DISCUSSION}

Figures 1 and 2 show experimental and simulated orthobaric densities for different alkenes up to 1-octene; propene, 1-butene, and 1-pentene in Fig. 1, and, 1-hexene and 1-octene in Fig. 2. In both figures, the open circles correspond to experimental data, and the filled circles are results from simulations with the current model. To complete the coexistence curves near the critical point, we fit the results of 


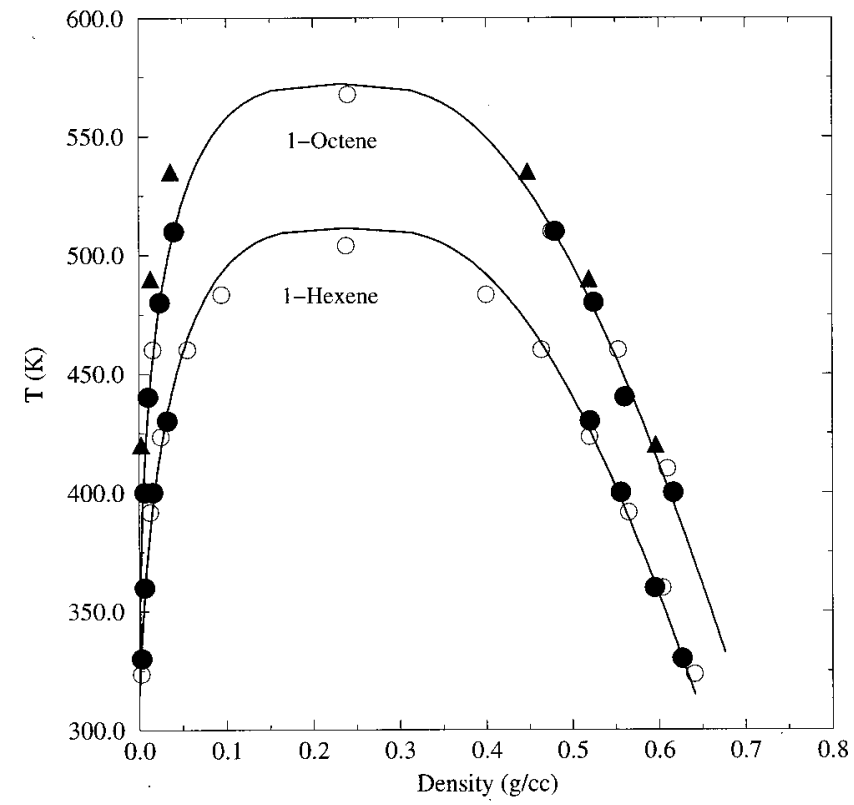

FIG. 2. Orthobaric densities for intermediate alkenes. The meaning of the symbols and lines is as in Fig. 1. Experimental data are taken from Ref. 18.

simulations to a simple Ising scaling-law analysis using a critical exponent $\beta=0.32 ;^{16}$ as we are mainly interested in the phase behavior of alkenes away from the critical point, no crossover or finite-size corrections were implemented in this work to estimate critical properties. The Ising fit curves are also shown in Figs. 1 and 2 by solid lines. Figures 1 and 2 show that simulation results for the alkenes are in good agreement with experimental data. Unfortunately, we cannot assess the accuracy of our force-field for phase equilibrium of longer alkenes (data are not available). For comparison, we also show predictions of the Spyriouni et al. model (using filled triangle symbols), in both Figs. 1 and 2. For 1-butene, Spyriouni et al. model underpredicts saturated liquid densities, and it gives a higher critical temperature. With increasing chain length, their predicted saturated liquid densities move to the right and overpredict the values for 1 -octene. Their model gives higher values of the critical temperature for all alkenes studied here.

Simulated coexistence densities and their statistical accuracies for the alkenes from the current model are given in Table III. Average densities are determined by constructing histograms from which the most probable value and the half width at half peak height are extracted.

An important test for molecular models is their ability to correctly describe the vapor pressure. Figure 3 shows simulated and experimental vapor pressures of 1-butene, 1-hexene, and 1-octene at several temperatures. Agreement between experiment and simulation is satisfactory.

As mentioned in the introduction, in this work we have also studied mixtures of alkenes with long alkanes. It is important to emphasize that one of our primary interests is to use force fields based on pure component properties to study various mixtures involving short and long olefins. In previous publications we have showed that this is possible for binary mixtures of short and long normal alkanes and
TABLE III. Simulation results for the orthobaric densities of alkenes.

\begin{tabular}{|c|c|c|c|}
\hline Alkene & $\begin{array}{c}T \\
{[\mathrm{~K}]}\end{array}$ & $\begin{array}{c}\rho_{\mathrm{vap}} \\
{[\mathrm{g} / \mathrm{cc}]}\end{array}$ & $\begin{array}{c}\rho_{\text {liq }} \\
{[\mathrm{g} / \mathrm{cc}]}\end{array}$ \\
\hline \multirow[t]{4}{*}{ Propene } & 300.0 & $0.024(2)$ & $0.494(7)$ \\
\hline & 280.0 & $0.015(2)$ & $0.524(6)$ \\
\hline & 260.0 & $0.008(1)$ & $0.553(5)$ \\
\hline & 230.0 & $0.00034(1)$ & $0.588(4)$ \\
\hline \multirow[t]{4}{*}{ 1-Butene } & 368.0 & $0.036(4)$ & $0.478(12)$ \\
\hline & 340.0 & $0.0204(3)$ & $0.523(8)$ \\
\hline & 317.0 & $0.014(2)$ & $0.558(6)$ \\
\hline & 290.0 & $0.0052(1)$ & $0.586(7)$ \\
\hline \multirow[t]{6}{*}{ 1-Pentene } & 430.0 & $0.062(8)$ & $0.448(21)$ \\
\hline & 410.0 & $0.044(6)$ & $0.487(19)$ \\
\hline & 380.0 & $0.0237(3)$ & $0.535(13)$ \\
\hline & 350.0 & $0.012(1)$ & $0.570(12)$ \\
\hline & 320.0 & $0.0086(1)$ & $0.599(9)$ \\
\hline & 290.0 & $0.0028(1)$ & $0.636(9)$ \\
\hline \multirow[t]{4}{*}{ 1-Hexene } & 430.0 & $0.0324(6)$ & $0.512(12)$ \\
\hline & 400.0 & $0.016(3)$ & $0.556(10)$ \\
\hline & 360.0 & $0.006(1)$ & $0.595(8)$ \\
\hline & 330.0 & $0.003(1)$ & $0.627(6)$ \\
\hline \multirow[t]{4}{*}{ 1-Octene } & 510.0 & $0.040(7)$ & $0.480(16)$ \\
\hline & 480.0 & $0.0235(3)$ & $0.525(12)$ \\
\hline & 440.0 & $0.01(2)$ & $0.5604(9)$ \\
\hline & 400.0 & $0.0055(1)$ & $0.616(8)$ \\
\hline
\end{tabular}

branched alkanes. ${ }^{8,9}$ Unfortunately, to the best of our knowledge, experimental data for mixtures with alkenes are limited; direct comparison of simulation results to experiments for mixtures was, therefore, minimal in this article.

For completeness, we study binary mixtures of 1-butene with hexatriacontane and we compare our results with the one experimental value available, ${ }^{20}$ as well as with the pre-

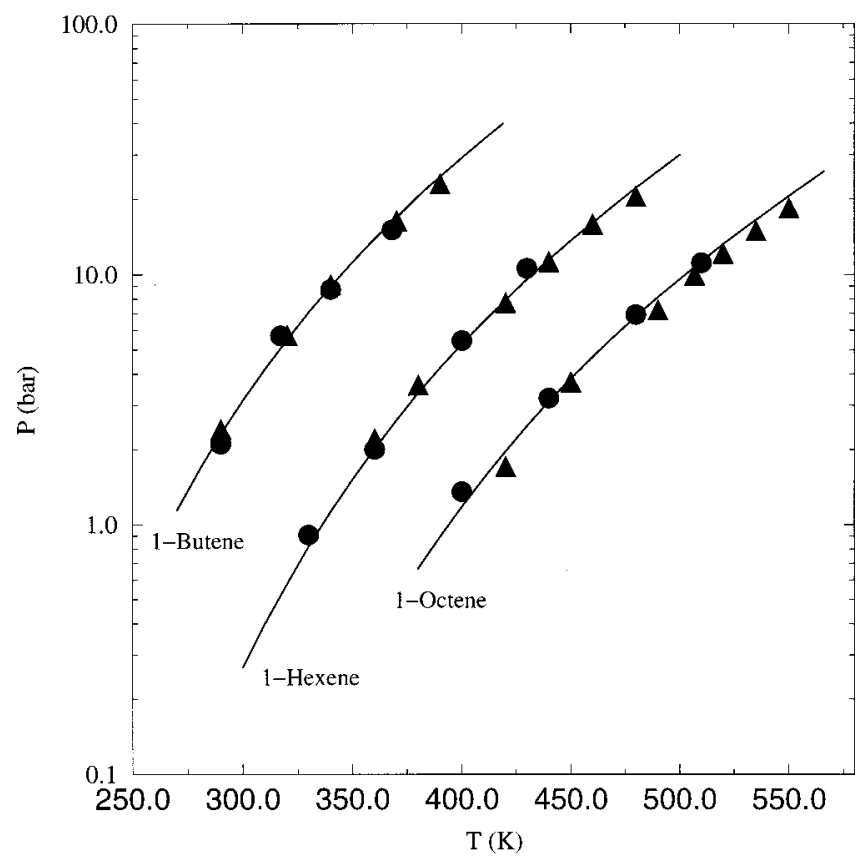

FIG. 3. Saturation pressures for 1-butene, 1-hexene, and 1-octene. The filled circles are simulation results from the present work, the filled triangles are from Spyriouni et al. (Ref. 12), and the curves are from experimental correlations (Ref. 19). The error bars for vapor pressure of the present work are about one symbol size. 


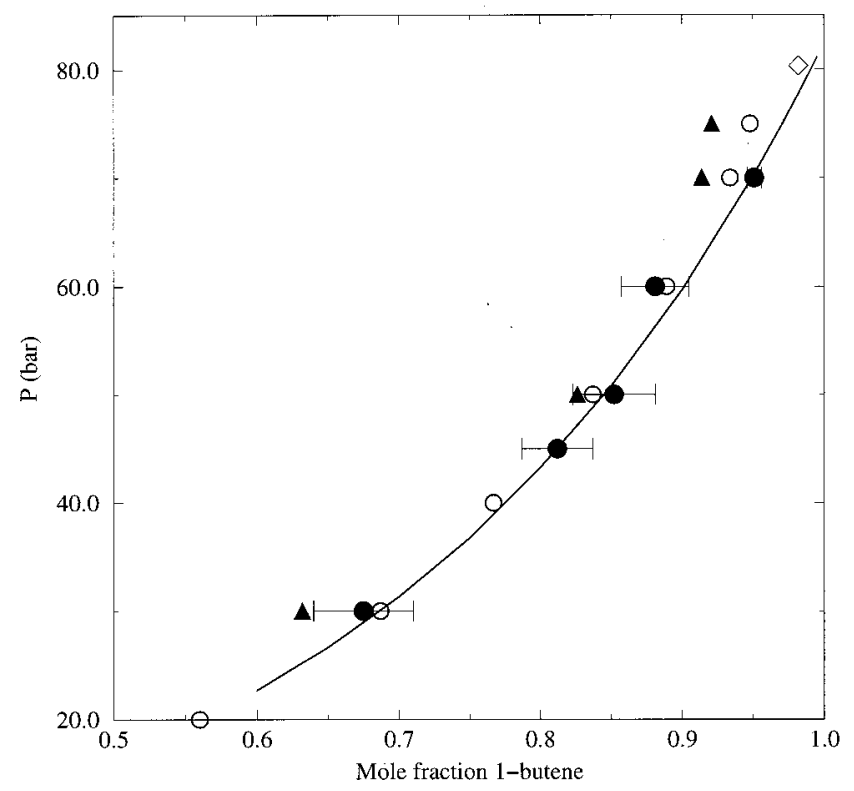

FIG. 4. Pressure vs composition diagram for a binary 1-butenehexatriacontane mixture at $T=473 \mathrm{~K}$. The filled circles are simulation results from present work, the filled triangles are from Spyriouni et al. (Ref. 12), open circles are predictions from SAFT, the open diamond is experimental (Ref. 20), and the curve is used as a guide to the simulation results of the present work. The error bars for our simulations are the standard deviation of block averages.

dictions of Spyriouni et al. and predictions from the statistical associating fluid theory (SAFT). ${ }^{21}$ The compressibility factor in SAFT is assumed to consist of a reference term and a polymer term which describes the effect of bonding of spherical sites to form chains. Detailed accounts of SAFT can be found in the original publication. ${ }^{21}$

Figure 4 shows a pressure vs composition diagram for a binary 1-butene-hexatriacontane mixture at $T=473 \mathrm{~K}$. Both simulations and SAFT calculations are conducted without the use of adjustable binary parameters. Our simulation results are in good agreement with the predictions from SAFT and the available experimental value; the pressure predictions from Spyriouni et al. are slightly higher. In previous work $^{5}$ we showed that SAFT predictions for phase equilibria in highly asymmetric mixtures (such as ethylene-eicosane, or ethylene-tetracontane) are not as accurate as those simulations with the NERD force field. Spyriouni et al. ${ }^{12}$ suggested that with increasing solvent size SAFT should be expected to become progressively better, which could explain why we see good agreement between SAFT and simulation predictions in this work.

Figure 5 shows results of simulations and SAFT predictions for the binary 1-butene-hexatriacontane system at constant pressure $(P=50 \mathrm{bar})$. This system shows evidence of a lower critical solution temperature (LCST). Simulation predictions from Spyriouni et al. are also shown in this figure. Our simulation results are slightly higher than those from the Spyriouni et al. model, and are similar to SAFT predictions. To the best of our knowledge, no experimental data are available for this case.

Density diagrams for both cases are shown in Figs. 6 and 7. Density results are compared to SAFT in both figures. For

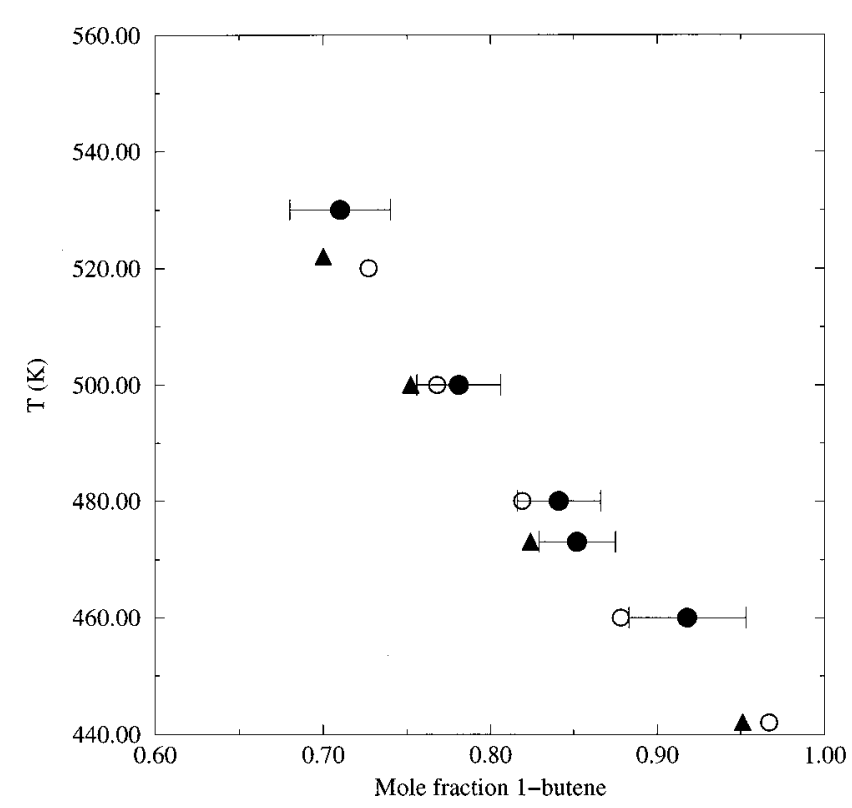

FIG. 5. Temperature vs composition diagram for a binary 1-butenehexatriacontane mixture at $P=50 \mathrm{bar}$. The meaning of the symbols are the same as in Fig. 4.

both systems, SAFT predictions are similar to our simulations. The slight deviations in density between simulation and SAFT are consistent with the slight deviations observed in the compositions of the systems. Unfortunately, no experimental data was found for the coexistence densities shown in Figs. 6 and 7.

\section{CONCLUSIONS}

In this manuscript, we have proposed a new set of united-atom force field parameters (NERD model) for

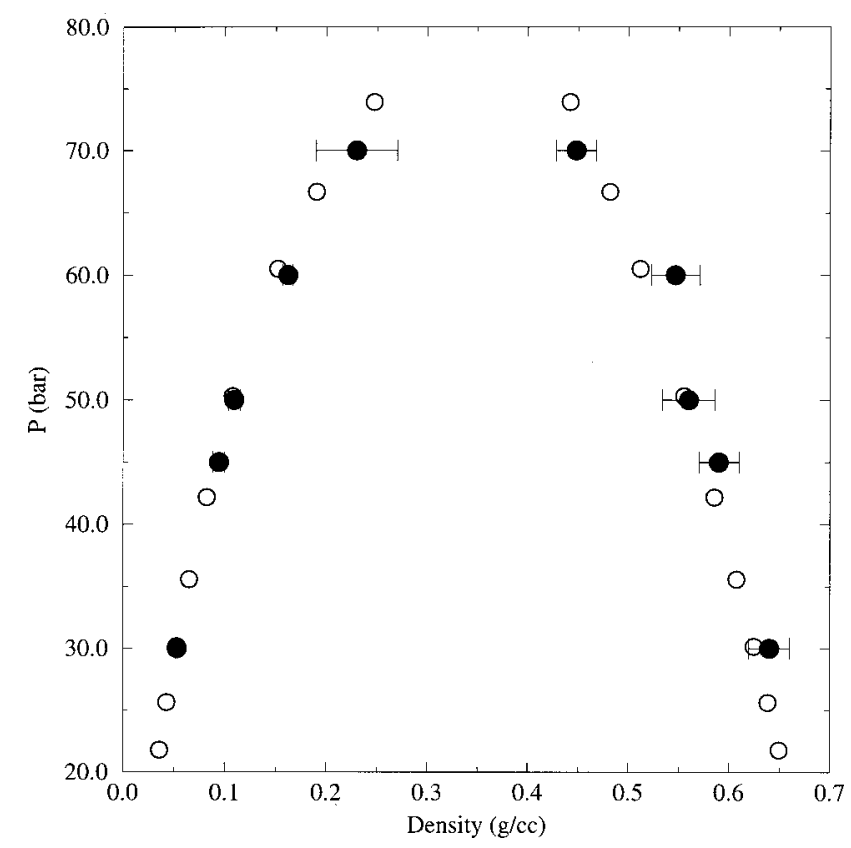

FIG. 6. Pressure vs density diagram for a binary 1-butene-hexatriacontane mixture at $T=473 \mathrm{~K}$. The filled circles are simulation results from present work, and open circles are predictions from SAFT. The error bars for our simulations are the standard deviaton of block averages. 


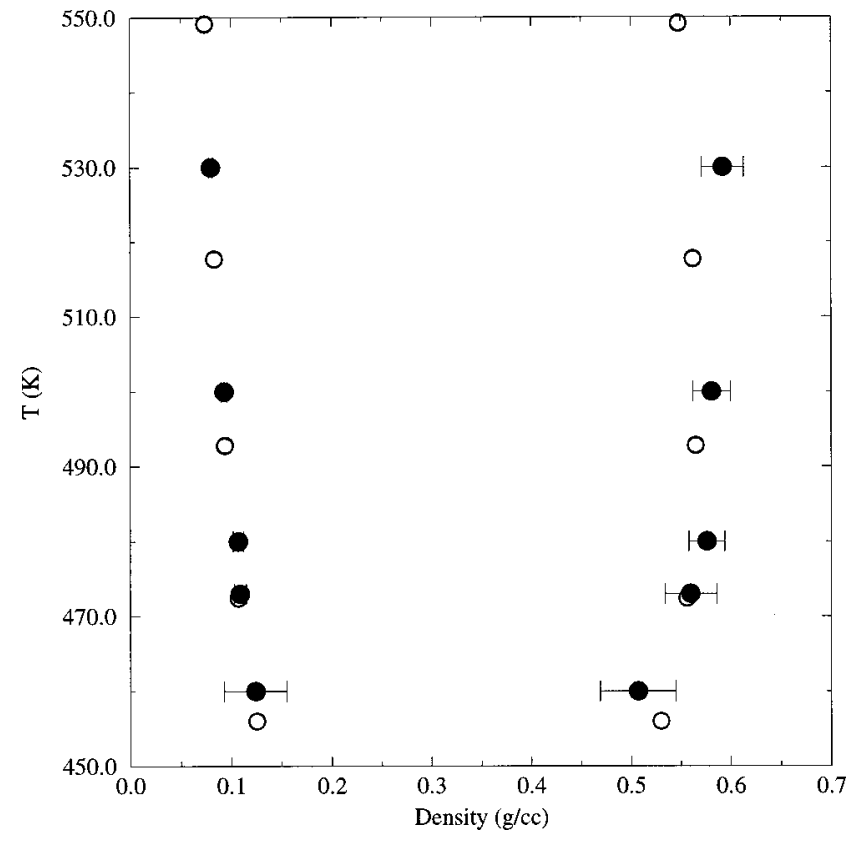

FIG. 7. Temperature vs density diagram for a binary 1-butenehexatriacontane mixture at $P=50$ bar. The meaning of the symbols are the same as in Fig. 6.

alkenes. The new force field is an extension of a previously proposed force field for linear alkanes and branched alkanes (NERD model). Good agreement with experimental coexistence properties was found for pure alkenes up to $C_{8}$. In the proposed model, end group $\mathrm{CH}_{2}\left(s p^{2}\right)$ parameters for ethylene and end group $\mathrm{CH}_{3}\left(s p^{3}\right)$ for propene are different than those for the rest of the alkene molecules.

Simulations were also conducted to study the ability of the proposed force field to describe low- and high-pressure phase equilibria in mixtures of small and long chain molecules. The systems studied here include mixtures of 1-butene and hexatriacontane. The results of simulations for mixtures were compared to predictions of the SAFT equation of state, predictions from the molecular model of Spyriouni et al., and one experimental datum; it was found that, for these mixtures, simulations are in quantitative agreement with the predictions from SAFT and are in agreement with the experimental datum.

\section{ACKNOWLEDGMENTS}

The authors wish to thank Bill Sachs and Sanjeev Rastogi of Union Carbide for helpful advice and discussions. This work was supported by the Division of Chemical Sciences, Office of Basic Energy Sciences, Office of Sciences, and U.S. Department of Energy. Acknowledgement is also made to the Donors of the Petroleum Research Fund, administered by the ACS, for partial support of this research.

${ }^{1}$ A. Z. Panagiotopoulos, Mol. Phys. 61, 813 (1987).

${ }^{2}$ M. Laso, J. J. de Pablo, and U. W. Suter, J. Chem. Phys. 97, 2817 (1992).

${ }^{3}$ G. C. A. M. Mooij, D. Frenkel, and B. Smit, J. Phys.: Condens. Matter 4, L255 (1992).

${ }^{4}$ F. A. Escobedo and J. J. de Pablo, J. Chem. Phys. 105, 4391 (1996).

${ }^{5}$ S. K. Nath, F. A. Escobedo, and J. J. de Pablo, J. Chem. Phys. 108, 9905 (1998).

${ }^{6}$ M. G. Martin and J. I. Siepmann, J. Phys. Chem. 102, 2569 (1998).

${ }^{7}$ J. R. Errington and A. Z. Panagiotopoulos, J. Phys. Chem. B 103, 6314 (1999)

${ }^{8}$ S. K. Nath and J. J. de Pablo, Mol. Phys. 98, 231 (2000).

${ }^{9}$ S. K. Nath, F. A. Escobedo, J. J. de Pablo, and I. Patramai, Ind. Eng. Chem. Res. 37, 3195 (1998).

${ }^{10}$ T. Spyriouni, I. G. Economou, and D. N. Theodorou, Phys. Rev. Lett. 80, 4466 (1998)

${ }^{11}$ W. L. Jorgensen, J. D. Madura, and C. J. Swenson, J. Am. Chem. Soc. 106, 6638 (1984).

${ }^{12}$ T. Spyriouni, I. G. Economou, and D. N. Theodorou, J. Am. Chem. Soc. 121, 3407 (1999).

${ }^{13}$ J. J. de Pablo, Q. Yan, and F. A. Escobedo, Annu. Rev. Phys. Chem. 50, 377 (1999).

${ }^{14}$ S. Duane, A. D. Kennedy, B. J. Pendleton, and D. Roweth, Phys. Lett. B 195, 216 (1987).

${ }^{15}$ S. K. Nath, J. J. de Pablo, and A. D. DeBellis, J. Am. Chem. Soc. 121, 4252 (1999).

${ }^{16}$ J. I. Siepmann, M. G. Martin, C. J. Mundy, and M. L. Klein, Mol. Phys. 90, 687 (1997).

${ }^{17}$ B. D. Smith and R. Srivastava, Thermodynamics Data for Pure Compounds: Part A Hydrocarbons and Ketones (Elsevier, Amsterdam, 1986).

${ }^{18}$ DIPPR Data Compilation of Pure Compound Properties, Design Institute for Physical Properties Data, American Institute of Chemical Engineers, 1987.

${ }^{19}$ R. C. Reid, J. M. Prausnitz, and B. E. Poling, The Properties of Gases and Liquids, 4th ed. (McGraw Hill, New York, 1987).

${ }^{20}$ C. J. Gregg, F. P. Stein, S. Chen, and M. Radosz, Ind. Eng. Chem. Res. 32, 1442 (1993).

${ }^{21}$ W. G. Chapman, K. E. Gubbins, G. Jackson, and M. Radosz, Fluid Phase Equilibria 52, 31 (1989); Ind. Eng. Chem. Res. 29, 1994 (1991). 